\title{
Revisión comentada de la política pública aplicada a la gestión de riesgo de desastres en la zona norte del páramo Cruz Verde, al oriente de Bogotá, Colombia
}

\author{
Commented review of public policy applied to \\ disaster risk management in the northern area of \\ the paramo Cruz Verde, east of Bogota, Colombia
}

María Alejandra Bermudez Ayala ${ }^{1}$

Fecha de recibido: 6 de mayo de 2021

Fecha de aceptado: 9 de julio de 2021

\section{Resumen}

El análisis de las diferentes amenazas y los factores de riesgo de desastres dentro de un territorio comprenden elementos fundamentales para el desarrollo en todos los ámbitos de una población, aborda la problemática desde los procesos de conocimiento y reducción del riesgo hasta la respuesta y recuperación ante un posible desastre. Este trabajo estudia las políticas públicas de gestión de riesgo en la parte norte del Páramo Cruz Verde ubicado al costado oriental de la ciudad de Bogotá. Para esto se revisan las propuestas del gobierno local en sus planes de gestión del suelo en esta zona, abarcando elementos que implementan las autoridades estatales en cuanto al ordenamiento territorial que delimitan las zonas en condición en riesgo y la presencia de eventos de amenaza en la región de estudio. Por consiguiente, es importante conocer cuáles son los entes reguladores que realizan estos estudios en Colombia y verificar sí las normativas se están realizando de

1 María Alejandra Bermudez Ayala, Universidad Distrital Francisco José Caldas, Colombia, correo electrónico: mabermudeza@correo.udistrital.edu.co. 
manera eficiente. Como parte de los resultados, se encuentran documentos normativos desactualizados e información geográfica, en algunos casos, no disponible al público. Adicionalmente, no existe una conexión estrecha entre las entidades científicas y administrativas que logre establecer un análisis interdisciplinar en el territorio. Por lo expuesto anteriormente, se recomienda realizar un diagnóstico detallado del riesgo para evitar la ocupación en terrenos no apropiados, y como parte de una oportunidad para el desarrollo local, ya que evita costosas inversiones que de una u otra manera los municipios deben sufragar en el momento de presentarse un desastre.

Palabras clave: geografía política, gestión de riesgos, páramo Cruz Verde, planificación territorial.

\section{Abstract}

Analysis of the different threats and disaster risk factors within a territory encompasses essential elements for development in all areas of a population. It addresses the problem from the processes of knowledge and risk reduction to the response and recovery in the event of a potential disaster. This paper studies the public policies of risk management in the northern part of the Paramo Cruz Verde located on the eastern side of the city of Bogotá. To this end, the proposals of the local government in their land management plans in this area are reviewed, covering elements implemented by the state authorities in terms of territorial planning that delimit the areas at risk and the presence of threat events in the study area. Therefore, it is important to know which regulatory bodies carry out these studies in Colombia and to verify that the regulations are being carried out efficiently. As part of the results, there are outdated policy documents and geographical information, in some cases, not publicly available. In addition, there is no close connection between scientific and administrative entities that can establish an interdisciplinary analysis in the territory. Based on the above, it is recommended that a detailed risk assessment be carried out in order to avoid occupancy of unsuitable land and as part of an opportunity for local development, since it avoids costly investments that one way or other municipalities have to pay for at the time of a disaster.

Key words: political geography, risk management, paramo Cruz Verde, territorial planning.

\section{Introducción}

Al realizar una mirada general hacia el territorio colombiano queda en evidencia el aumento de las cifras de zonas catalogadas en riesgo o que han tenido 
ocurrencia de un desastre por fenómenos de origen hidrometeorológicos. Además, la unidad técnica encargada de la Gestión del Riesgo de Desastres en Colombia afirma en su "Guía de Integración del Riesgo de Desastres en el Ordenamiento Territorial Municipal” que

...el riesgo de desastre se genera, en parte, cuando se ocupa un territorio, el cual, dadas las condiciones de amenaza presentes, no tiene la aptitud y/o capacidad para ser desarrollado o urbanizado, o sobre el cual no se definen las medidas de regulación necesarias respecto de la ocupación del suelo y no se ejecutan medidas de mitigación, o cuando en zonas ya ocupadas y construidas no se reconoce la existencia de una condición de riesgo y ésta no es controlada o reducida adecuadamente (Calderón et al., 2015, p. 3).

Cuando se analiza la importancia de la gestión de riesgo en los instrumentos de planeación, se establece un proceso disyuntivo como afirmó en 2016 Gustavo Adolfo Carrión en la unidad dos (2) del documento "Curso en gestión del riesgo de desastres para autoridades ambientales en Colombia”, el cual no se refiere únicamente a conceptos generales como territorio, desarrollo sostenible o planeación del desarrollo, más bien tiene que ver con el proceso participativo de toma de decisiones y con los beneficios o impactos resultantes. La integración de un marco adaptativo de gestión del riesgo resulta ser indispensable (Al-Amin et al., 2019) frente a estos temas de toma de decisiones.

La incorporación del riesgo en la planificación territorial es necesaria para determinar los tratamientos urbanísticos que se deberán implementar a fin de reducir el potencial de pérdidas de vidas y daños económicos en las zonas determinadas como de alto riesgo (Ministerio de Ambiente, Vivienda y Desarrollo Teritorial [MAVDT], 2005). Sin embargo, como se comenta en la Figura 1, existen vacíos en la gestión del riesgo:

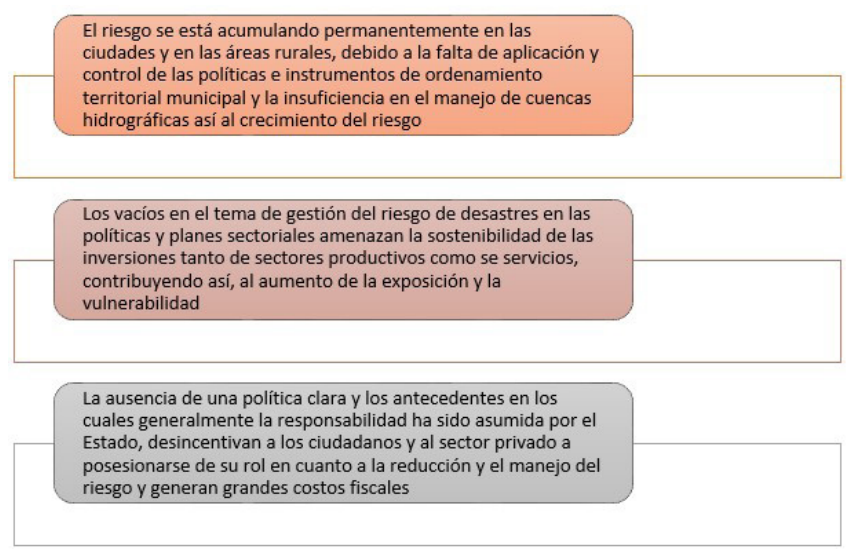

Figura 1. Visión general adoptada de la gestión de riesgo. 
Fuente: adoptado de MAVDT (2005).

La permanente intervención antrópica en los territorios y el constante deterioro ambiental, que tienen como consecuencia la pérdida de cobertura vegetal, la degradación de los suelos y la deforestación, ocasiona variaciones extremas en las condiciones climatológicas que desencadenan un aumento de los fenómenos amenazantes en el territorio, tales como inundaciones, avenidas torrenciales, entre otros. Lo anterior se suma a la "inadecuada articulación de las entidades y las debilidades en términos de armonización de los instrumentos de planificación territorial que inciden en la prevención del riesgo, pone en peligro a la población colombiana" (Castro et al., 2018, p. 12). De hecho, en la gestión del riesgo existen barreras que se relacionan con la falta de capacidades locales, la falta de apoyo político local y los desafíos tecnológicos en la fase de implementación (Thaler et al., 2019) que obstaculizan las iniciativas de adaptación en todo el mundo.

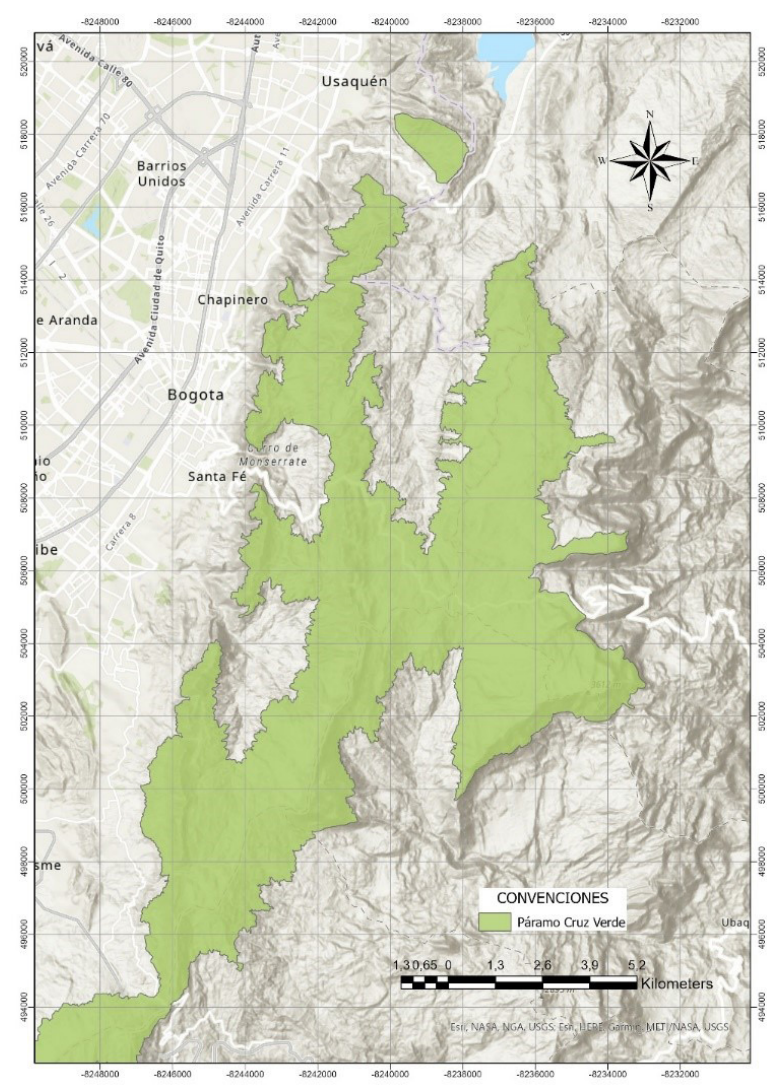

Figura 2. Zona de estudio norte del páramo Cruz Verde. 
Fuente: elaboración propia con datos tomados de la Gobernación de Cundinamarca y Datos abiertos de la Alcaldía de Bogotá (2020).

El páramo Cruz Verde resulta ser una muestra de esta problemática, sobre todo en la zona norte, como se observa en la Figura 2, donde se mantienen límites entre los municipios de Choachí, Chipaque y Ubaque, junto a las localidades de Chapinero, San Cristóbal y Santa Fe en Bogotá.

Con el desarrollo de este trabajo se busca realizar una revisión a los instrumentos de políticas públicas entorno a la gestión del riesgo en el territorio de estudio, generando conciencia para mejorar las condiciones del ecosistema y la calidad de vida de los habitantes aledaños; debido a que es posible la ocurrencia de riesgos de diferentes magnitudes que alteren la seguridad de la población, así mismo, encontrar las fortalezas y debilidades de las entidades encargadas de proteger la comunidad, el ecosistema y la riqueza ambiental en esta área.

Para ello, este artículo se ha dividido en tres partes. La primera, constituye un estudio con una mirada general frente al tema del conocimiento de riesgos. Para lo anterior es necesario conocer las instancias encargadas de la gestión de riesgo y establecer un estado del arte de las investigaciones que evalúan la gestión de riesgo en Latinoamérica y en Colombia. En la segunda parte se realiza una revisión comentada a partir de las políticas públicas relacionadas con la gestión de riesgo que se encuentran estipuladas en los municipios de Choachí, Ubaque y Chipaque, además de las localidades de Chapinero, San Cristóbal y Santa Fe, desde su marco normativo como la base de datos geográfica y cartográfica que zonifica el riesgo en la zona de estudio. Finalmente, la tercera parte hace referencia a la propuesta de priorización de escenarios de riesgo a partir de los marcos normativos anteriormente evaluados, con el fin de presentar eventos de desastre con mayor impacto alrededor de la zona norte del páramo Cruz Verde.

\section{Desde la política pública de gestión de riesgo}

Las discusiones sobre la organización del Estado, han estado presentes en buena parte de la historia político-institucional de Colombia, aunque desde una perspectiva generalmente administrativa y poco consecuente con procesos reales de conformación de asentamientos humanos, localización y dinámica de las actividades económicas, relaciones de movilidad, y presión de la población sobre ecosistemas regionales.

Sin embargo, las obligaciones de tratamiento de riesgo del gobierno a nivel nacional, departamental y municipal no son eximidas al crear instituciones administrativas públicas, pues la relación y el desarrollo de una buena gestión de riesgo en un territorio, depende fundamentalmente de las acciones y programas que incentiven los gobernantes, además de destinar 
tanto presupuesto como personal en todos los niveles con el fin de reducir el riesgo o disminuir la vulnerabilidad de los habitantes. "La articulación debe darse no sólo a través de la coherencia de los instrumentos sino a partir de la construcción de sinergias institucionales en los espacios e instancias de coordinación y participación de los sistemas territoriales y ambientales" (Carrión Barrero, 2016, p. 25).

Por lo anterior, a pesar de que la labor de gestión de riesgo dispone de autonomía en sus entidades territoriales, esto ha dado inicio a graves problemas de desactualización de la información y de pocos proyectos que mitiguen o prevengan una situación de amenaza en entidades territoriales que se caracterizan por una regular administración pública o que no disponen de herramientas que les ayude a realizar una gestión del riesgo, eficiente en su jurisdicción que integre a entidades públicas, a entidades privadas y a la comunidad.

Así mismo, en el país se considera la obligatoriedad a los municipios con la asistencia de las oficinas de planeación, de "levantar y mantener actualizado el inventario de las zonas que presentan altos riesgos para la localización de asentamientos humanos y adelantar programas de reubicación de los habitantes" (Instituto de Hidrología, Meteorología y Estudios Ambientales [IDEAM] y Programa de las Naciones Unidas para el Desarrollo [PNUD], 2014b, p. 8). Es necesario que en las revisiones de planes y políticas públicas se tenga en cuenta la interrelación de distintos conocimientos en todos los niveles estatales que atiendan de manera constante las problemáticas dentro de cada espacio geográfico y adopte medidas especiales con el fin de disminuir el riesgo al que pueden estar expuestos los actores integradores de dicho espacio.

Aun cuando la toma de decisiones es encabezada por el Consejo Nacional para la Gestión de Riesgo en asesoría de su cuerpo técnico en cada nivel territorial, se debe entender que se trata de una colaboración de agentes de administración pública, lo cual "indica que la planeación, ejecución, control y evaluación de los compromisos y acciones sobre reducción, conocimiento y manejo del riesgo requiere del entendimiento sobre el funcionamiento de sistemas de planeación y coordinación de acciones entre niveles territoriales y nacionales" (Carrión Barrero, 2016, p. 14).

Además de la descentralización de las funciones del Sistema Nacional de Riesgo es posible observar que existe un conjunto de entidades encargadas de aportar desde sus competencias y funciones procesos, proyectos o estrategias que vinculan todo lo relacionado con el conocimiento, reducción y tratamiento del riesgo que al final dan un resultado integrado del concepto y objetivos de la gestión del riesgo, y que "las competencias institucionales para su debida incorporación en los instrumentos de planificación ambiental y territorial no radican en cabeza de una única instancia o entidad sino de 
varias, de conformidad con sus facultades legales" (Carrión Barrero, 2016, p. 19). Por tanto, una mirada integral del riesgo (Figura 3) posibilita la capacidad de respuesta y desarrollo de la gestión del riesgo, de manera que todos los actores involucrados se beneficien y estimar la menor cantidad de daños en caso que se materialice un escenario de riesgo.

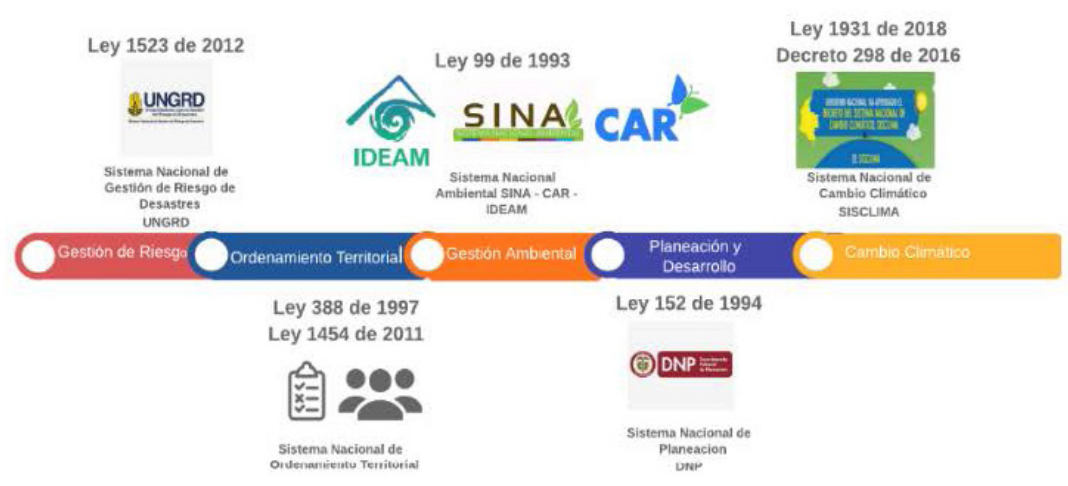

Figura 3. Abordaje preliminar de la gestión de riesgo a nivel nacional en Colombia. Fuente: Adaptado de MAVDT (2005); Unidad Nacional para la Gestión de Riesgos de Desastre (UNGRD 2012 y 2017).

\section{Estudios latinoamericanos}

Existen estudios y proyectos realizados en cuanto a reducciones de riesgos, por ejemplo el caso de la ciudad de Santo Domingo de Heredia de Costa Rica, donde hace algunos años el caudal del río Bermúdez sobrepasó los niveles normales y se desbordó causando grandes daños sobre el barrio Fátima en Santo Domingo, donde quedaron afectadas tanto las familias de esta población como sus viviendas; debido a esto los autores concluyen que son pocos los proyectos que se adelantaron en la ciudad y como consecuencia de la urbanización en zonas de alto riesgo se ha reflejado mala planeación de la ciudad con respecto a la expansión urbana por la que ha pasado los últimos años (Fernández et al., 2013).

Otro estudio es el de la mina Buenavista del Cobre, ubicada en Cananea, Sonora, México, donde ocurrió un derrame de sulfato de cobre acidulado en el río Sonora en 2014 (Aparicio et al., 2017). El derrame dio lugar a un desastre socio ambiental que afectó a los habitantes y a los ecosistemas de la cuenca del río. Los autores de este estudio afirman que:

...tras investigar cómo se gestionan los procesos de riesgo-desastre de origen antrópico, a partir del caso del derrame tóxico de la mina de cobre Buenavista, se encuentra que la política de protección civil y la ambiental, encargadas de estos 
riesgos, están desvinculadas entre sí, no convergen en objetivos, estrategias ni escalas de acción, lo que genera vacíos en su gestión, por lo que es necesaria una mayor interconexión entre las políticas, los niveles de gobierno, las empresas y la población para poder gestionar los riesgos químico- tecnológicos. Ambas políticas deben proyectarse sobre los planes de desarrollo local, estatal y nacional, con la finalidad de lograr una mayor congruencia entre el modelo de desarrollo y la protección de la población, sus bienes y los ecosistemas (p. 126).

Existe evidencia de casos donde la percepción de riesgo en la comunidad se encuentra afianzada al nivel de confianza en las autoridades locales (Hernandez y Ruiz, 2016) y eso ejemplifica la buena labor que, en algunas regiones como Teziutlán, México, se viene desarrollando para generar concertaciones equitativas con la comunidad.

Efectivamente, en América Latina algunos estudios frente a la implementación de la gestión social del riesgo son limitados y muchas veces no son incluidos en el ordenamiento territorial. Para Colombia desde la ocurrencia de los fenómenos de El Niño y La Niña a mediados del 2010 se han implementado normativas integrales frente a la mirada del proceso y tratamiento del riesgo.

\section{Estudios colombianos}

Después de los fenómenos de variaciones climáticas que desbordaron diferentes escenarios como inundaciones, se pone en marcha una iniciativa de abordar el riesgo de desastres por completo, desde la información y conocimiento de los posibles fenómenos del país hasta la preparación y respuesta cuando ocurra algún desastre. Se propuso una mayor destinación económica en los procesos de conocimiento y reducción del riesgo por desastre, lo cual amplió el campo de estudio frente a los escenarios de riesgo principales que se generan en Colombia, esto acompañado de instituciones y cargos especiales que acompañan y se encargan del componente de gestión de riesgo.

Cabe resaltar que algunos análisis de la gestión de riesgo originan estudios que se consideran recomendaciones para los instrumentos de ordenamiento territorial como el caso del proyecto de zonificación de Amenazas por Remoción en Masa, Sector La Nohora, Montecarlo al sur de la ciudad de Villavicencio, pues como conclusión, los autores Ortiz y Montón (2013) establecen que

A pesar del orden legal en materia de ordenamiento que está establecido para la zona estudiada y que define los términos de su conservación, se ha construido un ordenamiento, que aumenta la amenaza de un posible proceso de remoción 
en masa, en donde existe un alto grado de vulnerabilidad dada la densidad demográfica del asentamiento La Nohora (p. 11).

En el sector Isla La Manga del municipio de Yopal, Casanare se realizó la zonificación de la amenaza y vulnerabilidad por inundación (Arias, 2018). No obstante, entre las recomendaciones se invitan a los entes territoriales a mejorar su plataforma de datos geográficos y disponer la información a través de una infraestructura de datos espaciales, acorde a las políticas y normas internacionales.

Si se tiene en cuenta otros casos puntuales frente a estudios de gestión de riesgo en un territorio colombiano, se evidencia la falta de interrelación entre los escenarios de riesgos actuales con las políticas y los planes adoptados para reducir riesgos presentes, lo cual aumenta la vulnerabilidad en todas las áreas si se llega a materializar una amenaza. En Villavicencio (Ortega, 2014) se tuvo como resultado la siguiente reflexión después de un estudio en sus instrumentos de planeación territorial:

\begin{abstract}
Aunque el POT de Villavicencio, hace un intento por auto orientar sus directrices de ordenamiento, abarcando una visión integral del territorio, su competencia legal sigue estando limitada al ámbito municipal, por lo cual, no cuenta con un instrumento de escala territorial que lo oriente, que le sirva de guía para definir sus lineamientos, intenta hacerlo por sí mismo, pero se queda en un planteamiento abstracto que dista mucho de convertirse en un verdadero plan. Se podría decir entonces, que el único plan de escala superior que, en cierta medida, el POT toma como referencia para orientarse, es el Plan de Desarrollo Económico Departamental, del cual se revisan sus contenidos para detectar que aporta a la elaboración del plan (p. 45).
\end{abstract}

Este caso es evidencia de la falta de integración de las entidades ambientales, administrativas, de ordenamiento y de gestión de riesgos en el municipio, generando afectaciones en las medidas de reducción para riesgo presente o futuro que puedan minimizar los daños o pérdidas dentro de un municipio.

Por otro lado, existen estudios con información completa y actualizada, lo cual permite un desarrollo factible en caso de que se requiera conocer los riesgos en un territorio y promover proyectos que lo reduzcan a tiempo, donde los resultados encontrados fueron gracias a la inclusión de la gestión del riesgo dentro de los planes y esquemas de ordenamiento territorial, lo que permitió garantizar la identificación precisa de áreas críticas, donde no debe asentarse la población, ni realizar actividades que incrementen el riesgo de desastres (Calderón et al., 2015).

Adicionalmente, establecer nuevos proyectos de enfoque territorial como la cartografía resultante de uno de los estudios en Chía, permite obtener 
información fiable frente a fenómenos de remoción en masa, inundaciones e incendios, lo cual resulta de gran utilidad tanto en la fase de planificación de grandes obras públicas como en su fase de ejecución y a la hora de adoptar las oportunas medidas de prevención, mitigación y corrección (Burgos y Reina, 2017). El estudio en Chía señala que:

...los principales desafíos en los territorios, actualmente, señalan que los procesos de ordenamiento ambiental y territorial, deben articularse con los procesos de mitigación y adaptación al cambio y a la variabilidad climática; así como también, a los procesos de gestión del riesgo. Abordarlos desde un enfoque sinérgico, para que se logren materializar metas concretas de desarrollo sostenible, en cada porción específica de territorio. Teniendo en cuenta a la comunidad como eje, en las intervenciones que procedan, incrementando sus capacidades y el bienestar humano, con el fin de valorar y afrontar localmente los impactos de las cambiantes realidades del entorno (p. 98).

En el estudio de vulnerabilidad por avenidas torrenciales en Fusagasugá de William Andrés Salamanca Pira y Christian Andrés Torres Sarmiento, se menciona falta de comunicación de la comunidad vulnerable con la amenaza latente con la que convive, lo cual hace parte del proceso de conocimiento de riesgo, debido a que aseguran que

La presencia de las entidades municipales encargadas ha sido importante para la preparación de las familias, sin embargo, se necesita todavía de más intervención en capacitaciones ya que el porcentaje de familias que desconoce los pasos a seguir en una emergencia es todavía muy alto (Torres Sarmiento y Salamanca Pira, 2017, p. 111).

Lo anterior, en similitud a lo que ocurre en otras ciudades de Colombia, no obstante, para un municipio con menos recursos económicos es aún más desfavorable la situación.

Varios estudios parten de "la identificación de visiones divergentes sobre un mismo peligro, posibilita que los estudios de percepción social del riesgo pasarán a recibir la máxima atención como un posible enfoque de abordaje para explicar las causas de las diferencias" (Ferrari, 2012, p. 113). Cabe resaltar que el componente de vulnerabilidad en el proceso de identificar riesgos es sumamente relevante, pues es el factor que tiene influencia social y económica, demuestra la susceptibilidad de una población y, por tanto, García (1997) asegura que

...la cohesión social se muestra como otro de los factores que componen la vulnerabilidad, de tal modo que las sociedades que poseen una trama compleja de organizaciones pueden absorber más fácilmente las consecuencias de un desastre y reaccionar con mayor rapidez que las que no la tienen. La cohesión 
social puede manifestarse tanto formalmente, a través de organizaciones oficiales o sectores de la administración pública especializados en el tema, como de forma espontánea entre grupos que por diversas razones se involucran en estas cuestiones. Las estrategias de prevención y mitigación de los efectos de una catástrofe que puedan establecerse y el análisis de su eficacia es un campo poco explorado todavía (p. 13).

Para este trabajo es fundamental abordar los procesos de conocimiento y de reducción del riesgo como ejes integradores de una óptima disminución de daños y pérdidas de vida. Por consiguiente, se debe hacer una mirada detallada a cada uno de los instrumentos que integran la gestión del riesgo en el ordenamiento del territorio y evaluar la conexión entre ellos que faciliten el intercambio de conocimientos y generen en conjunto planes, proyectos y soluciones orientadas a mejorar la calidad de vida de los habitantes a partir de reducir el riesgo en cada entidad territorial. La importancia de este proyecto radica que en gran parte de los estudios acerca de la gestión de riesgo se ha generado una desarticulación entre la teoría de gestión de riesgo y las políticas o lineamientos (Calderón et al., 2015) que en la práctica se aplican frente al acontecimiento de un desastre.

\section{Compendio de los instrumentos públicos para atender la gestión de riesgo}

El análisis de los instrumentos consta de un conjunto de planes y documentos para estipular los componentes de conocimiento, reducción y manejo de desastre dentro del proceso de gestión de riesgo (Figura 4). En la "Guía de Integración de la Gestión del Riesgo y el Ordenamiento Territorial Municipal" desarrollada por la Unidad Nacional para la Gestión de Riesgo de Desastres en 2015, están establecidos los determinantes en los estudios de riesgo de desastres en el ordenamiento territorial y sustentados en la Ley 388 de 1997 se encuentran (Calderón et al., 2015):

- La definición de políticas, directrices y regulaciones sobre la identificación y prevención de amenazas y riesgos.

- El señalamiento y localización de áreas de riesgo para la localización de asentamientos humanos.

- La definición de estrategias de manejo de áreas expuestas a amenazas y riesgos naturales.

- La definición de zonas no urbanizables que presenten riesgo para la localización de asentamientos humanos, por amenazas naturales y socionaturales.

- La determinación de las áreas con condición de amenaza o con condición de riesgo. 
- La determinación de la mitigabilidad o no mitigabilidad del riesgo.

- La determinación de áreas de riesgo no mitigable y definición de estas áreas como suelos de protección.

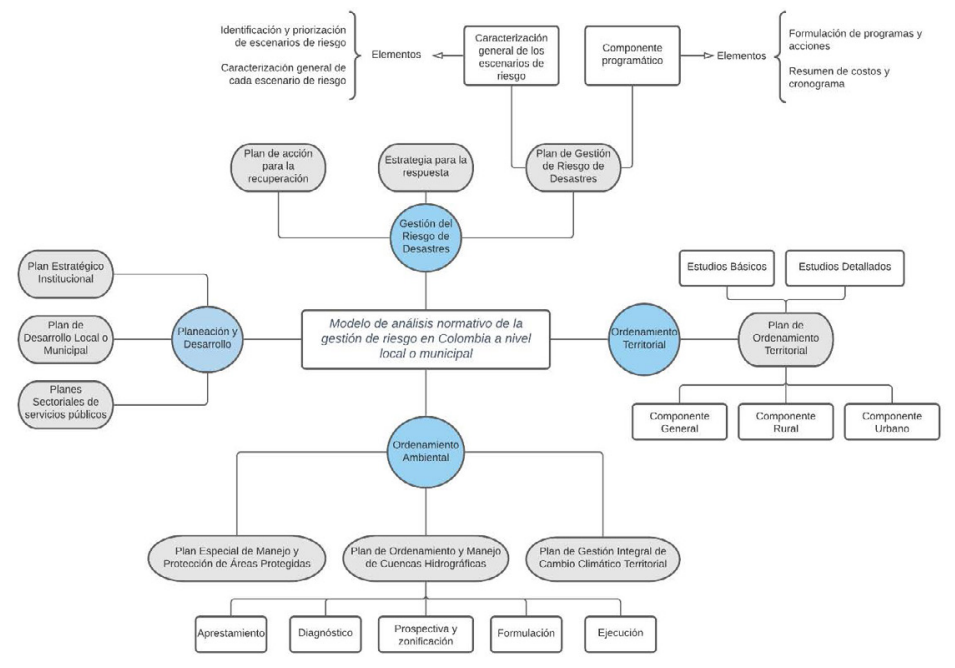

Figura 4. Propuesta modelo de análisis normativo de la gestión de riesgo en Colombia a nivel local o municipal.

Fuente: adoptado de IDEAM y PNUD (2014a).

- La localización de áreas críticas de recuperación y control para la prevención de desastres.

\section{Revisión comentada del estado de los instrumentos y diagnóstico normativo de la zona de estudio}

Al observar el caso de los instrumentos de planeación territorial en el páramo Cruz Verde zona nororiental de la ciudad de Bogotá, aunque la vigencia actual de actualización catastral es del 2018, el último Esquema de Ordenamiento Territorial (EOT) de Choachí es del año 2000 cuando se expidió el Acuerdo 005 del mismo año que adoptó el instrumento, por tanto, la información no se puede tener en cuenta cuando se requiere evaluar la situación actual de los riesgos en el municipio.

Para el caso Chipaque se adopta el EOT por medio del Acuerdo 011 del 2000 y se prevé un desarrollo a mediano plazo hasta el 2005 y a largo plazo hasta el 2010. Sin embargo, hasta la fecha siguen las autoridades territoriales en el proceso de actualización del EOT, en el caso de Ubaque se adopta el EOT por el Acuerdo 001 del 2000 y hasta la fecha se ha realizado una propuesta de actualización con una vigencia catastral del año 2005 pero no se ha concretado 
tal actualización del instrumento, además su información cartográfica sigue las recomendaciones que expidió INGEOMINAS en 1995.

Por consecuente, la información mínima de los EOT que establece el Decreto 1077 de 2015 que corresponde a análisis detallado de amenaza, evaluación de vulnerabilidad, evaluación del riesgo y determinación de medidas de mitigación, se encuentra limitada para los municipios que comprenden la zona de estudio.

Cada municipio cuenta con las siguientes instancias de dirección:

- Consejo Municipal para la Gestión de Riesgo y Desastres (CMGRD)

- Plan Municipal de la Gestión de Riesgo y Desastres (PMGRD)

- Estrategia Municipal de Respuesta a Emergencia (EMRE)

- Fondo Municipal de Gestión de Riesgo de Desastres (FMGRD).

Sin embargo, la información de éstas no se encuentra abierta ni es de fácil acceso a la comunidad o a personas interesadas, los temas relacionados con Gestión del Riesgo no se abarcan en la totalidad del municipio.

La información de gestión de riesgo en los Esquemas de Ordenamiento Territorial de los municipios se encuentra desactualizada y sin la respectiva información histórica de eventos presentados. Por lo contrario, las localidades de Bogotá que forman parte del área de estudio promueven programas y establecen planes de gestión de riesgos como la caracterización de escenarios de riesgo, planes locales de gestión del riesgo y cambio climático, y diferentes estrategias. También, se encuentran algunas dinámicas, propuestas y planes dentro del POT de Bogotá, pero de manera muy generalizada. Cada localidad cuenta con la siguiente información actualizada dentro de los Planes Locales de Gestión del Riesgo y Cambio Climático (PLGR-CC):

\section{PLGR-CC Chapinero}

1. Caracterización de Escenarios de Riesgo

a. Identificación y Priorización

b. Escenario de Riesgo por Movimientos en Masa

c. Escenario de Riesgo por Incendios Forestales

d. Escenario de Riesgo por Accidentes de Tránsito

e. Escenario de Riesgo por Encharcamiento e Inundaciones

2. Componente Programático

a. Componente programático

b. Acciones a corto plazo

3. Adopción del Plan Local de Gestión del Riesgo y Cambio Climático

a. Acto administrativo de adopción PLGR/CC

\section{PLGR-CC Santa Fe}

4. Caracterización de Escenarios de Riesgo 

a. Identificación y Priorización
b. Escenario de Riesgo por Movimientos en Masa
c. Escenario de Riesgo por Incendios Forestales
d. Escenario de Riesgo por Siniestros Viales

5. Componente Programático
a. Componente programático
b. Acciones a corto plazo

6. Adopción del Plan Local de Gestión del Riesgo y Cambio Climático

a. Acto administrativo de adopción PLGR/CC

\section{PLGR-CC San Cristóbal}

7. Caracterización de Escenarios de Riesgo
a. Identificación y Priorización
b. Escenario de Riesgo por Aglomeración de Público
c. Escenario de Riesgo por Incendios Forestales
d. Escenario de Riesgo por Avenida Torrencial
e. Escenario de Riesgo por Movimientos en Masa
f. Escenario de Riesgo por Elementos Expuestos en Establecimientos Educativos
g. Escenario de Riesgo por Accidentes de Tránsito
h. Escenario de Riesgo Sanitario

8. Componente Programático
a. Componente programático
b. Acciones a corto plazo

9. Adopción del Plan Local de Gestión del Riesgo y Cambio Climático

a. Acto administrativo de adopción PLGR/CC

También se presenta cartografía de las zonas de riesgo en los instrumentos de planeación territorial para todo el borde de estudio. Sin embargo, la información no está al día y no se encuentra al nivel de detalle que ordena la norma, pues según el Decreto 1077 de 2015 cuando se realizan los estudios básicos para la formulación de los EOT se requieren escalas 1:25 000 en suelos rurales y 1:5000 en suelos urbanos y para los estudios detallados los cuales se implementan directamente a los EOT la escala para suelo urbano es de 1:2 000 y de suelo rural de 1:5000. Por tanto, al verificar los productos cartográficos las escalas exceden el límite de la norma prevista para estudios tanto en las fases de formación como implementación de los Esquemas de Ordenamiento Territorial.

Algunos factores climáticos que deben incluirse para estudios de zonas de amenaza se presentan de manera muy general, dichos estudios son indispensables por la disposición de información de las condiciones 
atmosféricas y físicas de la zona. Teniendo en cuenta que estos estudios hacen parte del factor fundamental para el estudio físico y la planificación del territorio, por lo que su no realización afecta a la gestión del riesgo de desastres.

En los EOT de los tres municipios no se encuentra información actualizada con respecto a geomorfología de la zona, siendo ésta fundamental en estudios del medio físico y análisis de riesgos. Sin embargo, los Planes de Desarrollo se encuentran actualizados para cada uno de los municipios debido a que con cada cambio de gobierno es necesario la expedición de estos planes, el problema con ello es que no hay concordancia con los otros instrumentos de planeación y por tanto en el caso de establecer proyectos, programas 0 normativas en relación con la gestión de riesgo, no hay mucha factibilidad en desarrollarlos.

Lo importante para resaltar es la labor de las entidades públicas de investigación y de registro que contienen información actualizada de la cual se pueden desprender estudios para cada uno de los municipios y que se espera que sean acogidos para la formulación de los nuevos EOT.

Por otro lado, la Ley 1523 de 2012 promueve el desarrollo de proyectos de entidades públicas que se relacionen con la gestión del riesgo y sirvan de insumo para la elaboración de normativas y documentos técnicos que deben emitir las entidades territoriales. Es por eso que las Corporaciones Autónomas Regionales, el Instituto de Hidrología, Meteorología y Estudios Ambientales (IDEAM), del Servicio Geológico Colombiano (SGC), Instituto Alexander Von Humboldt, Departamento Administrativo Nacional de Estadística (DANE), el Departamento Nacional de Planeación (DNP), entre otros; incluyen información sobre cada uno de los componentes de la gestión del riesgo a lo largo del territorio colombiano.

Frente a los niveles de vulnerabilidad, amenaza y riesgo de desastres en los instrumentos de planeación territorial se sectorizan algunas zonas con mayor prioridad, sin embargo, la información no parece estar al día y deja muchos vacíos frente a la situación del municipio.

Como alternativa a lo anterior, en el 2018 el Departamento Nacional de Planeación generó un análisis del riesgo de cada municipio ajustado por capacidades que vincula todos los posibles escenarios de riesgo. El índice se imparte a través de la evaluación separada del componente de Riesgo y del componente de Capacidades.

Para el riesgo se evaluó la amenaza de las áreas municipales con las condiciones más críticas en las que pueden presentarse inundaciones lentas, flujos torrenciales y movimientos en masa donde los resultados arrojaron que la región andina abarca la mayor zona de amenaza. También, se evaluó la exposición donde el departamento con mayor incidencia fue Cundinamarca 
y por último se evaluó la vulnerabilidad, la cual se midió a través del Índice de Pobreza Multidimensional (IPM), cuyas dimensiones reflejan la limitación en oportunidades que tienen los hogares para acceder y movilizar activos para gestionar el riesgo. En el caso del componente de Capacidades se abarcó la variable financiera, la de gestión de riesgo y la socioeconómica (Departamento Nacional de Planeación [DNP], 2018). Los resultados se pueden apreciar en la Tabla 1 para la zona de estudio de este trabajo:

Tabla 1. Índice de riesgos ajustado por capacidades

\begin{tabular}{|c|c|c|c|c|}
\hline Municipio & Chipaque & Ubaque & Choachí & Bogotá \\
\hline $\begin{array}{l}\text { Exposición proporción área } \\
\text { amenazada }\end{array}$ & $90,5 \%$ & $75,8 \%$ & $81,8 \%$ & $24,9 \%$ \\
\hline Vulnerabilidad (IPM Ajustado) & $28,5 \%$ & $25,9 \%$ & $20,0 \%$ & $26,7 \%$ \\
\hline Índice de Riesgo & 25,77 & 19,59 & 16,38 & 6,66 \\
\hline Ranking índice de riesgo & 390 & 539 & 630 & 930 \\
\hline $\begin{array}{l}\text { Estado del Comité Municipal } \\
\text { de Gestión de Riesgo de } \\
\text { Desastre (CMGRD) }\end{array}$ & $\begin{array}{l}\text { No } \\
\text { reportado }\end{array}$ & Creado & Creado & $\begin{array}{l}\text { No } \\
\text { reportado }\end{array}$ \\
\hline $\begin{array}{l}\text { Estado del Plan Municipal } \\
\text { de Gestión de Riesgo de } \\
\text { Desastre (PMGRD) }\end{array}$ & Formulado & $\begin{array}{l}\text { No } \\
\text { reportado }\end{array}$ & Formulado & Adoptado \\
\hline $\begin{array}{l}\text { Estado de la Estrategia } \\
\text { de Manejo de Respuesta } \\
\text { a Emergencias (EMRE) }\end{array}$ & $\begin{array}{l}\text { No } \\
\text { reportado }\end{array}$ & $\begin{array}{l}\text { No } \\
\text { reportado }\end{array}$ & $\begin{array}{l}\text { No } \\
\text { reportado }\end{array}$ & Adoptado \\
\hline Dimensión financiera & 0,42 & 0,17 & 0,25 & 0,95 \\
\hline Dimensión socioeconómica & 0,18 & 0,23 & 0,23 & 0,64 \\
\hline $\begin{array}{l}\text { Dimensión de gestión del } \\
\text { riesgo de desastres }\end{array}$ & 0,07 & 0,31 & 0,29 & 0,68 \\
\hline Índice de capacidades & 22,21 & 25,71 & 23,48 & 75,86 \\
\hline $\begin{array}{l}\text { Índice de riesgo ajustado } \\
\text { por capacidades }\end{array}$ & 55,3 & 51,3 & 49,9 & 30,6 \\
\hline $\begin{array}{l}\text { Ranking Índice de Riesgo } \\
\text { ajustado por capacidades }\end{array}$ & 389 & 557 & 618 & 1118 \\
\hline
\end{tabular}

Fuente: adoptado de DNP (2018).

Según el Plan Distrital de Gestión de Riesgos y Cambio Climático para Bogotá D.C., 2015-2050, los estudios detallados de amenaza y riesgo realizados por el IDIGER entre 1999 y 2010 corresponde para la localidad de Chapinero y Santa Fe 
a 6 estudios y para la localidad de San Cristóbal es de 32 estudios. Así el número de obras realizadas por el IDIGER entre 1998 y 2008 frente a riesgo por remoción en masa corresponde de la siguiente manera: 7 para la localidad de Chapinero, 14 para la localidad de Santa Fe y 28 para la localidad de San Cristóbal.

En Bogotá los planes de desarrollo hablan de la importancia de proyectos enfocados a la gestión de riesgo, sin embargo, se queda corto en temas de reasentamientos ya que no propone metas a cumplir ni horizontes de cantidad de familias reubicadas, ni cómo se recuperará este territorio. También, es importante resaltar que las alcaldías locales dejan el tema de gestión del riesgo y cambio climático a los consejos locales de gestión de riesgo y cambio climático, sin embargo, se pueden generar problemas en caso de que el Distrito decida declarar emergencia de cambio climático. Por tanto, las políticas y recursos de las alcaldías locales se deberán encaminar a dar solución o minimizar la problemática ambiental, así como, desastres en cada uno de los fenómenos que representen amenaza.

Un aspecto importante para mencionar es que los instrumentos territoriales tales como: POMCAs del Rio Palmar o del Rio Blanco, Documentos técnicos de la Corporación Autónoma Regional CAR y Documentos de Parques Naturales Nacionales, que corresponden a la extensión Páramo Cruz Verde dentro de la zona de estudio, evidencian de forma completa las características, los conjunto de recursos naturales que posan en la zona, las existentes actividades ganaderas y agrícolas que posiblemente infieren en el desarrollo de deslizamientos, mencionan además, actividades antrópicas que a lo largo del tiempo han aumentado como la explotación minera. Es importante tener en cuenta que la vinculación de información de estas entidades con los instrumentos territoriales es escasa (Rubio, 2008). En el caso de los municipios al lado oriental del Páramo Cruz Verde podrían vincular los datos que brindan las entidades ambientales para la reforma de los EOT.

\section{Escenarios de riesgos priorizados}

En la priorización de los escenarios de riesgo, la "Guía para la integración de la gestión de riesgo de desastre en los planes de desarrollo territorial 2020-2023" estipula que se debe tener en cuenta (Rivera y Manrique, 2020):

- Los escenarios de riesgo que puedan afectar la infraestructura esencial: hospitales, clínicas o centros de salud, aeropuertos, estaciones de transporte, centros logísticos humanitarios.

- Los escenarios de riesgo que tengan un número mayor de elementos y población vulnerable, es decir que representen un alto nivel de pérdidas potenciales.

- Los escenarios de riesgo que represente ante la ocurrencia de un fenómeno amenazante la pérdida de vidas humanas. 
- Los escenarios de riesgo que puedan representar mayores costos de recuperación.

De acuerdo con la anterior revisión en los instrumentos de planificación, se obtiene información de entidades ambientales para aproximar los escenarios de riesgo con mayor impacto (Figura 5): Riesgo por Movimientos en Masa e Incendios Forestales tienen mayor ocurrencia y afectación en la zona del Borde Oriental que comprende los municipios de Choachí, Ubaque, Chipaque y las localidades de Chapinero, Santa Fe y San Cristóbal. Esto se debe a la revisión en los documentos públicos (Figura 4) tales como Esquemas de Ordenamiento Territorial, Planes de Gestión de Riesgos, además de distintas informaciones

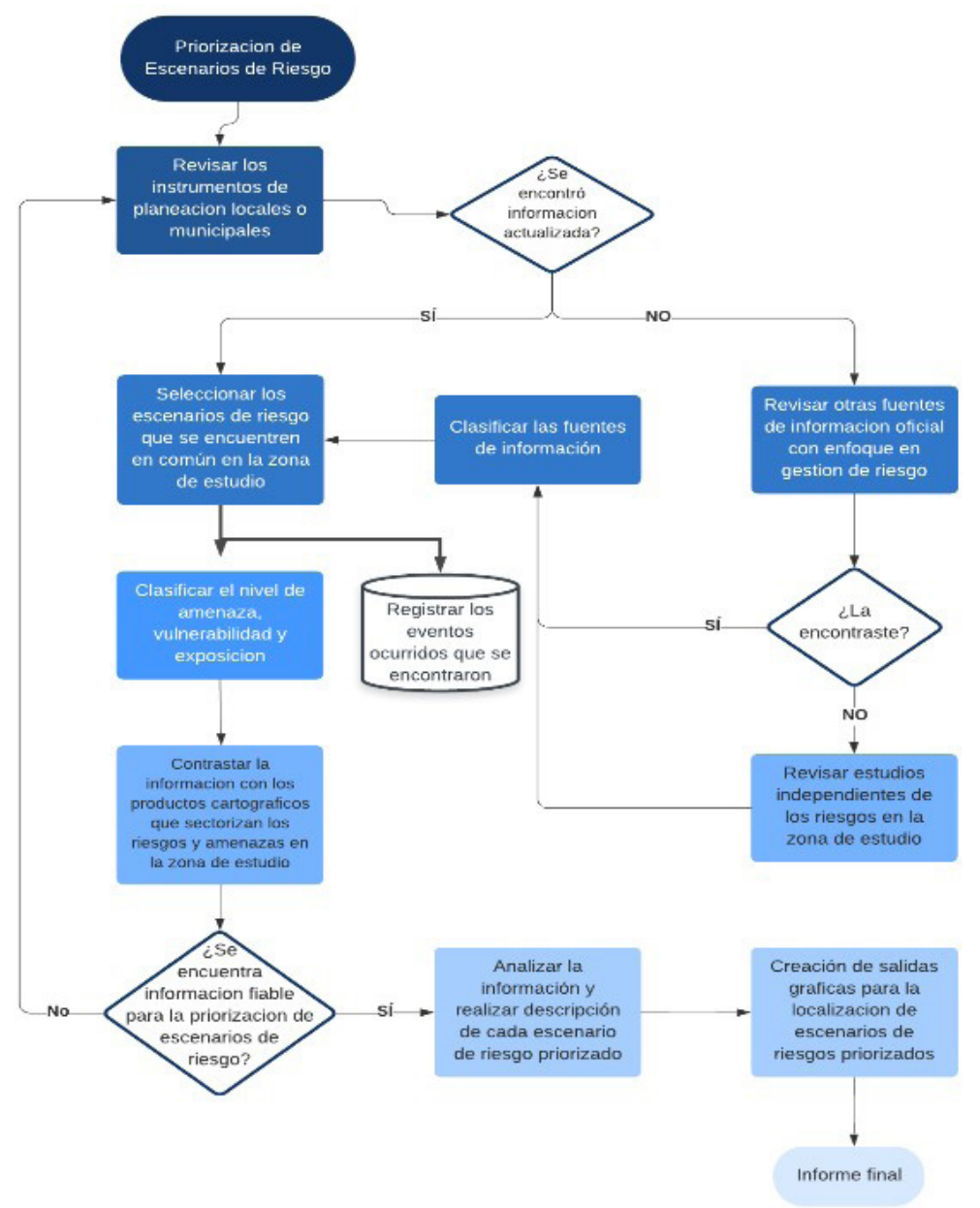

Figura 5. Propuesta de modelo de priorización de escenarios de riesgo en la zona de estudio. 
Fuente: elaboración propia.

oficiales como documentos de la Corporación Autónoma Regional, soportes en el registro y bases de datos del Departamento de Bomberos en Bogotá, del Instituto de Hidrología, Meteorología y Estudios Ambientales, del Servicio Geológico Colombiano, Departamento Administrativo Nacional de Estadística (DANE) y el Departamento Nacional de Planeación (DNP).

Los datos a continuación (Tabla 2) corresponden a una recopilación aproximada del número de eventos de Incendios Forestales y Movimientos en Masa desde el año 1998 hasta el 2017:

Tabla 2. Eventos de desastres registrados entre 1998 y 2017

\begin{tabular}{ccc}
\hline \multirow{2}{*}{ Evento } & Ubicación & $\begin{array}{c}\text { Eventos registrados } \\
(1998-2017)\end{array}$ \\
\hline \multirow{4}{*}{$\begin{array}{c}\text { Movimientos } \\
\text { de masa }\end{array}$} & Municipio Choachí & 7 \\
\cline { 2 - 3 } & Municipio Ubaque & 6 \\
\cline { 2 - 3 } & Municipio Chipaque & 8 \\
\cline { 2 - 3 } & Localidad Chapinero & 130 \\
\cline { 2 - 3 } Incendio & Localidad San Cristóbal & 414 \\
\cline { 2 - 3 } forestal & Municipio Choachí & 185 \\
\cline { 2 - 3 } & Municipio Ubaque & 29 \\
\cline { 2 - 3 } & Municipio Chipaque & 20 \\
\cline { 2 - 3 } & Localidad Chapinero & 270 \\
\cline { 2 - 3 } & Localidad San Cristóbal & 650 \\
\cline { 2 - 3 } & Localidad Santa Fe & 220 \\
\hline
\end{tabular}

Fuente: Adoptado de datos de la Unidad Administrativa Especial de Gestión de riesgos de desastres (UAEGRD, 2017); Sistema de Información para la Gestión del Riesgo y Cambio Climático SIRE (2018); Instituto Distrital de Gestión de Riesgos y Cambios Climáticos (IDIGER, 2019).

De acuerdo con reportes de autoridades ambientales como el Instituto de Hidrología, Meteorología y Estudios Ambientales (IDEAM), el Servicio Geológico Colombiano (SGC), el Instituto Distrital de Gestión de Riesgos y Cambio Climático (IDIGER) (datos del sistema de alerta de Bogotá [SIRE] 2002-2014), las entidades de primeros auxilios como la Unidad Administrativa Especial cuerpo oficial Bomberos de Bogotá y los Planes de Gestión de Riesgo en las localidades, es posible aproximar los focos de concentración de ambos escenarios de riesgo priorizados con mayor impacto generado en la zona de estudio. 


\section{Reflexiones finales}

En la zona norte del páramo Cruz Verde se encuentran falencias en cuanto a la incorporación de gestión y manejo de riesgo en las herramientas de ordenamiento territorial. Para hacer un análisis real del riesgo hay que empezar a contextualizar los instrumentos gubernamentales y entender la ineficiencia al invertir en obras de infraestructura de manejo y mitigación del riesgo si no se tiene en cuenta las dinámicas poblacionales, históricas y ambientales para las que se está gestionando el riesgo. Por ejemplo, uno de los problemas en la zona de borde son los asentamientos ilegales, lo cual se presenta en todos los municipios y las localidades; generan un cambio en el ecosistema que finalmente se convierte en riesgo con el tiempo.

Los instrumentos de gestión de riesgos de la zona de estudio promueven programas para la prevención y mitigación del riesgo, sin embargo en algunos casos se encuentran desactualizados y no permiten, como en el caso de los EOT, tener una evolución más dinámica ya que su periodo de renovación es de 12 años y como en el caso de Bogotá hasta de 15 años, en este tiempo se pueden presentar fenómenos que obliguen a reformular y actualizar estos instrumentos, por ejemplo no mantener actualizados los instrumentos de ordenamiento según los contextos actuales hacen que las acciones sean retrasadas o retrogradas, lo que obstaculiza el salvamento de vidas y las acciones encaminadas a este objetivo.

En el caso del área de estudio, aunque en las entidades territoriales existan herramientas normativas, el contenido en algunas no es de acceso público o por lo general se encuentran desactualizadas. Por lo cual, es necesario un diagnóstico detallado para identificar de forma anticipada las zonas donde se puede generar riesgo, lo que resulta fundamental para determinar correctamente las áreas de expansión del municipio a fin de evitar desastres futuros.

Para la adecuada formulación de los planes de desarrollo departamentales y municipales, la gestión del riesgo de desastres se constituye como un eje estructurante para garantizar la sostenibilidad territorial, y debe ser concebida por los alcaldes y gobernadores como una herramienta para orientar los adecuados procesos de desarrollo. Es decir, para alcanzar la sostenibilidad y la construcción segura del territorio, es fundamental el reconocimiento de la gestión del riesgo de desastres como un condicionante para las futuras inversiones y debe estar sujeta a un ejercicio de planificación, donde las autoridades, a partir del conocimiento de las áreas de amenaza y áreas de riesgo, tengan las herramientas suficientes para la toma de decisiones, y evitar así, las inversiones en zonas de amenaza y el aumento de las condiciones de riesgo presentes en territorio. 
Es importante entender que la gestión de riesgo requiere de una mirada transversal en todos los instrumentos de planeación, que la labor no solo corresponde a las entidades públicas ambientales, sino se requiere que dentro de cada jurisdicción el gobierno mantenga actualizada la información y destine recursos públicos en la generación de productos detallados de cada escenario de riesgo que afecta la calidad de vida de las personas, pues aunque existan estudios y datos registrados, es a través de los instrumentos de planificación que se puede concretar ayudas en cada región. Finalmente, se incentiva la inclusión ciudadana para quienes se encuentran habitando el territorio y generar conocimiento del riesgo para evitar futuros asentamientos en la zona del páramo.

\section{Referencias}

Al-Amin, A. Q., Nagy, G. J., Masud, M. M., Leal Filho, W., \& Doberstein, B. (2019). Evaluating the impacts of climate disasters and the integration of adaptive flood risk management. International Journal of Disaster Risk Reduction, 39, 101241. https://www.doi.org/10.1016/j.ijdrr.2019.101241.

Alcaldía de Bogotá (2020). Plataforma distrital de Datos Abiertos. https://datosabiertos.bogota.gov.co/

Alcaldía de Choachí (2000). El Esquema de Ordenamiento Territorial (EOT) de Choachí. Cundinamarca, Colombia

Alcaldía de Chipaque (2000). El Esquema de Ordenamiento Territorial (EOT) de Chipaque. Cundinamarca, Colombia.

Alcaldía de Ubaque (2000). El Esquema de Ordenamiento Territorial (EOT) de Ubaque. Cundinamarca, Colombia.

Alcaldía Mayor de Bogotá (2015). DC Secretaría Distrital de Ambiente. Instituto Distrital de Gestión de Riesgos y Cambio Climático IDIGER (2015-2050). Plan Distrital de Gestión de Riesgos y Cambio Climático para Bogotá DC, 2050.

Alcaldía Mayor de Bogotá, Gobernación de Cundinamarca, Parques Nacionales Naturales de Colombia (2014). Incorporación de la gestión de riesgos hidroclimáticos en el ordenamiento territorial municipal.

Andrade, A., \& Amaya, M. (1994). El ordenamiento territorial en el Instituto Geográfico "Agustín Codazzi": aproximación conceptual y metodológica. Instituto Geográfico Agustín Codazzi, Proyecto SIG-PAFC. Revista SIG-PAFC, 1(3), $32-46$.

Aparicio, A. T., \& Hernández Canales, P. de J. (2017). Gestión de riesgos y desastres socioambientales. El caso de la mina Buenavista del cobre de Cananea. Investigaciones Geográficas, Boletín del Instituto de Geografía, (93), 126-139. https://doi.org/10.14350/rig.54770

Arias, J. (2018). Zonificación de la amenaza y vulnerabilidad por inundación, mediante modelamiento espacial en el sector isla la Manga municipio de Yopal, Casanare. Universidad Distrital Francisco José de Caldas. http://repository.udistrital.edu.co/handle/11349/14685 
Burgos Galindo, A. L., \& Reina Cuervo, S. X. (2017). Análisis de los escenarios de riesgo por fenómenos amenazantes para el municipio de Chía Cundinamarca como herramienta de planificación territorial [Tesis de Grado, Universidad Distrital Francisco José de Caldas]. http://hdl.handle.net/11349/4584

Calderón, J. M. S., Pérez, C. I. M., \& Hernández, R. A. V. (2015). Guía integración de la Gestión del Riesgo de Desastres y el Ordenamiento Territorial Municipal. Unidad Nacional de Gestión de Riesgo de Desastres, República de Colombia.

Carrión Barrero, G. A. (2016). Curso en Gestión del Riesgo de Desastres para Autoridades Ambientales en Colombia. Programa de las Naciones Unidas para el Desarrollo (PNUD) 30.

Carrión Barrero, G. A. (2008). Debilidades del nivel regional en el ordenamiento territorial colombiano. Aproximación desde la normatividad política administrativa y de usos del suelo. Centre de Política del Sòl i Valoracions - Universitat Politècnica de Catalunya (Eds.). https://doi.org/10.5821/ace.v3i7.2444

Castro, T., del Pilar, N., \& Peñaloza Molina, L. K. (2018). Detección y análisis de deslizamientos utilizando interferometría diferencial (D-Insar) entre los años 2015 y 2017 en las provincias norte y valderrama del departamento de Boyacá Colombia. Monografía, Universidad Distrital Francisco José de Caldas. http://hdl.handle.net/11349/13777

Consejo Local de Gestión de Riesgos y Cambio Climático (CLGR-CC) (2019). El Plan Local de Gestión del Riesgo y Cambio Climático PLGR-CC. Localidad de Chapinero. Bogotá, Colombia.

Consejo Local de Gestión de Riesgos y Cambio Climático (CLGR-CC) (2017). Caracterización General de Escenarios de Riesgo. Localidad de Santa Fe. Bogotá, Colombia.

Consejo Local de Gestión de Riesgos y Cambio Climático (CLGR-CC) (2017). Caracterización General de Escenarios de Riesgo. Localidad de San Cristóbal. Bogotá, Colombia.

Corredor de Ecosistemas Estratégicos de la Región Central de la Cordillera Oriental, (2015). Estudios técnicos, económicos, sociales y ambientales para la identificación y delimitación del complejo de páramos de Cruz-VerdeSumapaz a escala 1:25 000. Documento de caracterización del entorno regional, CEERCCO, Bogotá.

DNP (Departamento Nacional de Planeación) (2018). Índice municipal de riesgo de desastres ajustado por capacidades. Bogotá D.C., Colombia.

Fernández, M., Borges, J., Meléndez, G., Mora, F., Mora, J., \& Muñoz, C. (2013). Análisis de gestión de riesgo de inundación en la ciudad de Santo Domingo de Heredia. Costa Rica en el tercer milenio: desafíos y propuestas para la reducción de vulnerabilidad ante los desastres, San José.

Ferrari, M. P. (2012). Análisis de vulnerabilidad y percepción social de las inundaciones en la ciudad de Trelew, Argentina. Cuadernos de Geografía. Revista Colombiana de Geografía, 21(2), 99-116.

García Tornel, F. C. (1997). Algunas cuestiones sobre geografía de los riesgos. Scripta Nova, Revista Electrónica de Geografía y Ciencias Sociales, 10, 165-178. 
Gobernación de Cundinamarca. (2020). Portal de Datos Abiertos. Promoción de los datos y definición del Ecosistema de Datos Abiertos para Colombia. https://mapasyestadisticas-cundinamarca-map.opendata.arcgis.com/

Hernandez, Bertha, \& Ruiz, Naxhelli (2016). The production of vulnerability to landslides: the risk habitus in two landslide-prone neighborhoods in Teziutlán, Mexico. Investigaciones geográficas, (90), 7-27. https://doi.org/10.14350/rig.50663

Hernández Peña, Y. T. (2010). El ordenamiento territorial y su construcción social en Colombia: ¿un instrumento para el desarrollo sustentable? Cuadernos de Geografía. Revista Colombiana de Geografía, (19), 97-109. https://doi.org/10.15446/rcdg.n19.16854

Hernández Peña, Y., \& Vargas Cuervo, G. (2015). "Hacia la construcción de conocimiento emergente para la gestión local del riesgo". Cuadernos de Geografía. Revista Colombiana de Geografía, 24(2), 15-34. http://10.15446/rcdg.v24n2.50204

Highland, L. M., \& Bobrowsky, P. (2008). Manual de derrumbes. Guía pare entender todo sobre los derrumbes. Reston, Virginia, Circular 1325 del Sistema Geológico de los EUA, $129 \mathrm{pp}$.

Instituto de Hidrología, Meteorología y Estudios Ambientales y Programa de las Naciones Unidas para el Desarrollo (2014a, abril). Compilación y análisis de información sobre registros de eventos de emergencia y desastre asociados al clima en la región capital 1980-2010. Plan Regional Integral de Cambio Climático. Región Capital Bogotá - Cundinamarca. http://www.ideam.gov.co/documents/40860/609198/INFORME+TECNICO_ Evento+de+emergencias+y+desastres.pdf/e6fe9be7-1085-4736-9f899eb7e9fa0274? version $=1.0$

Instituto de Hidrología, Meteorología y Estudios Ambientales y Programa de las Naciones Unidas para el Desarrollo (2014b, abril). Piloto de asistencia técnica para incorporar la gestión integral de riesgos hidroclimáticos en el ordenamiento territorial municipal. Plan Regional Integral de Cambio Climático. Región Capital Bogotá. https://oab.ambientebogota.gov.co/wpcontent/uploads/dlm_uploads/2018/11/informe-tecnico_piloto-ot.pdf

Instituto Alexander Von Humboldt. (2017). Recomendación para la delimitación, por parte del Ministerio de Ambiente y Desarrollo Sostenible, del Complejo de Páramos de Sumapaz - Cruz Verde a escala 1:25.000. Bogotá: Instituto de Investigación de Recursos Biológicos Alexander Von Humboldt - Fondo Adaptación.

Instituto Distrital de Gestión de Riesgos y Cambio Climático IDIGER. (2019) Proyecto actualización de componente de gestión del riesgo para la revisión ordinaria y actualización del Plan de Ordenamiento Territorial Documento Técnico de Soporte Mapa de amenza por incendios forestales escala 1:25000.

Mashi, S. A., Oghenejabor, O. D., \& Inkani, A. I. (2019). Disaster risks and management policies and practices in Nigeria: A critical appraisal of the National Emergency Management Agency Act. International Journal of Disaster Risk Reduction, 33, 253-265. 
MAVDT (Ministerio de Ambiente, Vivienda y Desarrollo Territorial) (2005). Guía metodológica para incorporar la prevención y la reducción de riesgos en los procesos de ordenamiento territorial. Serie Ambiente y Desarrollo Territorial, Guía 1. Dirección de Desarrollo Territorial - DDT. Bogotá D.C.

Ortega Hernández, S. M. (2014). Análisis de la insuficiencia de los instrumentos de planeamiento en la escala territorial en la Ley 388 de 1997: El caso de Villavicencio, Colombia [Tesis de maestría en Gestión y valoración urbana, Universitat Politècnica de Catalunya]. http://hdl.handle.net/2099.1/24419

Ortiz, J. F. H., \& Montón, G. C. (2013). Zonificación de amenazas por remoción en masa, sector La Nohora-Montecarlo: Recomendaciones al plan de ordenamiento territorial POT. Cap\&Cua Ciencia, Tecnología y Cultura, 10(1), 1-12.

Pérez, M. V. (2010). El ordenamiento territorial en Colombia a través del tiempo. Perspectiva Geográfica. Revista del Programa de Estudios de Posgrado en Geografía, (15), 143-156.

Thaler, T., Attems, M. S., Bonnefond, M., Clarke, D., Gatien-Tournat, A., Gralepois, M., \& Servain, S. (2019). Drivers and barriers of adaptation initiatives-How societal transformation affects natural hazard management and risk mitigation in Europe. Science of the total environment, 650, 1073-1082. https://doi.org/10.1016/j.scitotenv.2018.08.306

Torres Sarmiento, C. A., \& Salamanca Pira, W. A. (2017). Análisis de la vulnerabilidad por avenidas torrenciales en la Vereda Pekín y el casco urbano del Municipio de Fusagasugá [Tesis de grado, Universidad Distrital Francisco José de Caldas]. http://hdl.handle.net/11349/5307

Rivera Gutiérrez, D. A., \& Manrique Osorio, J. D. (2020). Guía para la integración de la gestión del riesgo de desastres en los planes de desarrollo territorial 2020-2023.

Rubio, P. (2008). Los suelos del transecto Sumapaz (Cordillera oriental colombiana). En Van der Hammen, T. (Ed.), La Cordillera Oriental Colombiana Transecto Sumapaz. Estudios de Ecosistemas Tropandinos Vol. 7, 59-142.

Secretaría Distrital de Planeación SDP (2013). Documento Técnico de Soporte. Modificación Excepcional del Plan de Ordenamiento Territorial.

SIRE (Sistema de Información para la Gestión del Riesgo y Cambio Climático) (2018). Instituto Distrital de Gestión de Riesgos y Cambio Climático. Alcaldía Mayor de Bogotá. https://www.sire.gov.co/

Unidad Administrativa Especial de Gestión de Riesgos de Desastre (UAEGRD) (2017). Análisis Departamental de Gestión de Riesgo de Desastres de la Gobernación de Cundinamarca. Gobernación de Cundinamarca.

Unidad Nacional de Gestión de Riesgos de Desastre (UNGRD) (2017). Terminología sobre Gestión del Riesgo de Desastres y Fenómenos Amenazantes 2017. Documento elaborado por Comité Nacional para el Conocimiento del Riesgo. Comisión Nacional Técnica Asesora para el Conocimiento del Riesgo.

Unidad Nacional de Gestión de Riesgos de Desastre (UNGRD) (2012). Formulación del Plan Municipal de Gestión del riesgo (versión 1). Programas de reducción de la vulnerabilidad fiscal del Estado frente a desastres naturales. Banco Mundial. 